Inge Hinterwaldner

\title{
Erlebnis-Raum in Der Garten und Heufieber
}

Zusammenfassung: Normalerweise erfolgt die Situierung des Betrachters zur gebotenen Bildwelt über die Perspektivierung, beispielsweise durch die Zentralperspektive, die dem Publikum einen bestimmten Blickpunkt zuweist. Mit zeitbasierten Arbeiten ist es hingegen möglich, entweder die Perspektivierung als eine dynamisierte zu exemplifizieren oder Veränderungen des Blickpunktes über die Art der gezeigten bildlich-motivischen Dynamik in der Rezeption zu provozieren. Der Beitrag zielt darauf $a b$, verschiedene Weisen der Verschränkung zwischen Perspektivierung und Bildzeitlichkeit zu thematisieren. Die künstlerische Animation Der Garten macht mit bildnerischen Mitteln erfahrbar, dass eine intensive Auseinandersetzung mit der Umwelt letztere für die Tätigen je unterschiedliche Qualitäten annimmt. Zur Verdeutlichung wird der Darstellungsraum mit den Handlungen der Protagonistin korreliert und damit auch dynamisiert. Die zweite besprochene Arbeit, das Video Heufieber, funktioniert insofern analog, als dass das im ersten Beispiel gezeigte Motiv einer Weltgestaltung nun in die Rezeption verlagert ist und somit als Erfahrung nicht mehr für andere externalisiert wird. Der antizipierte aktive Betrachter moduliert seinen Raumeindruck aufgrund der gesammelten Erfahrungswerte angesichts von Abläufen. Um das komplexe Phänomen zu umkreisen, werden theoretische Positionen aus unterschiedlichsten Gebieten herangezogen: Über die Beobachtung (zweiter Ordnung) und Niklas Luhmann lässt sich Handeln und Betrachten verschränken, mit Henri Bergson wird die qualitative Auffassung von Raum denkbar und schließlich bietet Ludwig Wittgenstein neben der Gestaltpsychologie über den Aspektwechsel ein theoretisches Instrumentarium, um die festgestellten Bildraumumschwünge in Heufieber zu charakterisieren.

\section{Einleitung}

Am Beispiel zweier künstlerischer Arbeiten wird versucht, >Welt‘ über das Konzept des >Raums her zu fassen, oder genauer darüber, dass man sich in und zu einer Welt auf mehrfache Weise positionieren kann. In diesem Kontext wird eine bildliche Darstellung ${ }^{1}$ (von Raum) dann als Welt im Kleinen

1 Auch wenn dieser Beitrag eine Videoaufzeichnung und eine computergenerierte Animation (mit digitalisiertem Filmmaterial) ins Zentrum stellt, werden die Erzeugnisse dieser Techniken als >Bilder verstanden und unter bildlichen Vorzeichen diskutiert. Mit Gottfried Boehm lassen sich visuelle Konstellationen dann als >Bilder auffassen, wenn eine zweifache ikonische Differenz gegeben ist: als Unterschied zwischen Umgebung und dem fraglichen Artefakt einerseits und als optische Binnenmarkierungen, die in der Rezeption realisiert werden, andererseits (vgl. Boehm 1994, bes. 29-31; vgl. ferner Müller 1997). Selbstverständlich führen das reproduzierende Medium >Video< und das synthetische Verfahren 
angesprochen, wenn sie es erlaubt, sich auf mehr als eine Art dazu zu verhalten bzw. zu stellen. Oder anders gewendet: Bilder weisen dann Aspekte von sWelt auf, wenn man ihnen gegenüber nicht nur - wie üblich - eine einzige Haltung sinnvoll einnehmen kann, sondern wenn sie mehrfache Situierungen und damit Raumauffassungen zu erfahren gestatten. Hier stehen folglich weniger die Eigenschaften der von Bildern erzeugten Welten im Zentrum als Bilder, die zeigen, wie eine Welterzeugung (als räumliche Vielfalt) funktionieren kann. Die Erklärung des Mechanismus des wechselnden Raumeindrucks als Zusammenspiel eines identisch oder relativ stabil bleibenden Sehangebots mit der Rezeption hat Psychologen wie Philosophen schon des Längeren beschäftigt. Während die sogenannten Kippbilder zwei Weisen der Auslegung erlauben, weist das hier besprochene zeitbasierte Beispiel Heufieber aufgrund seiner manifesten Bewegung mehrere Wechsel und zudem neue Merkmale auf.

\section{Ansichten aus dem Garten}

Die Computeranimation Der Garten. Ein Amateurfilm des 21. Jahrhunderts (1992) des ungarischen Künstlers Tamás Waliczky zeigt seine eineinhalbjährige Tochter im Garten. Sie erkundet dort engagiert die Wiese, riecht an Blumen, stellt Insekten nach, umarmt einen Pfosten, setzt sich unter einen Baum, spießt Blätter auf, hängt sich an eine Schaukel, erklimmt die Leiter der Rutschbahn, um sodann die Talfahrt anzutreten. Im Zentrum steht die große Aufmerksamkeit und Entdeckerfreude, mit der ein Kleinkind seine Umwelt inspiziert. Das nachmittägliche Herumtollen wurde an einem einzigen Tag filmisch aufgenommen. ${ }^{2}$ Aus dem einstündigen Rohmaterial wählte der Künstler gut vier Minuten für die Animation aus, digitalisierte die Sequenzen und bearbeitete sie erheblich nach, um den gewünschten Ausdruck zu erzielen. Frame für Frame schnitt er das Kind per Hand der Silhouette nach aus, um die gefilmte Umgebung durch eine computergenerierte zu ersetzen. Mit dieser Maßnahme gewann er die be-

>Animation< jeweils unterschiedliche Konnotationen mit sich. Im konstruktiven Prinzip der Perspektivierung treffen sich beide Darstellungstechniken in den vorgestellten Beispielen. Einerseits wäre ohne aufwändigste Berechnungen und Überarbeitungen des bildlichen Rohmaterials in Der Garten das Zeigen der dynamischen Perspektive des Kindes nicht möglich, und ohne eingebaute automatisierte Perspektivierung ist andererseits keine Kamera denkbar: weder eine animationstechnisch nachempfundene noch eine reale. Bei der handelsüblichen - an die Zentralperspektive angelehnten - Aufzeichnungslogik des von Signer verwendeten Videogerätes sticht dann aber umso stärker ins Auge, dass trotz dieser gleichbleibenden perspektivischen Fixierung sich Bildelemente vor der Kameralinse so organisieren ließen, dass die hier thematisierte Wandlung im Kopf des Betrachters erfolgen kann.

2 Dennoch sind Zeitsprünge dadurch angedeutet, dass das Mädchen aufgrund einer Retusche mehrmals die Kleidung gewechselt zu haben scheint: Mal ist sie komplett in Gelb gekleidet, dann in Rosa, oder sie kombiniert einen weißen Pulli mit rosa Latzhose. Vermutlich ist der Farbwechsel bei jedem Schnitt - übrigens auch den Hintergrund betreffend - ein Mittel, um die Möglichkeiten der neuen Technologie auszuloten bzw. sich nicht an die Beschränkungen von Aufzeichnungsmedien halten zu müssen, sondern die Farbkomposition in jeder Szenerie neu aufeinander abzustimmen. 
nötigte Flexibilität der Darstellung. Denn die in jeder Film- oder Videokamera inhärente Aufzeichnungslogik wollte der Künstler abstreifen, um die Welt des Kindes von dessen Warte aus wiederzugeben (Abb. 1).

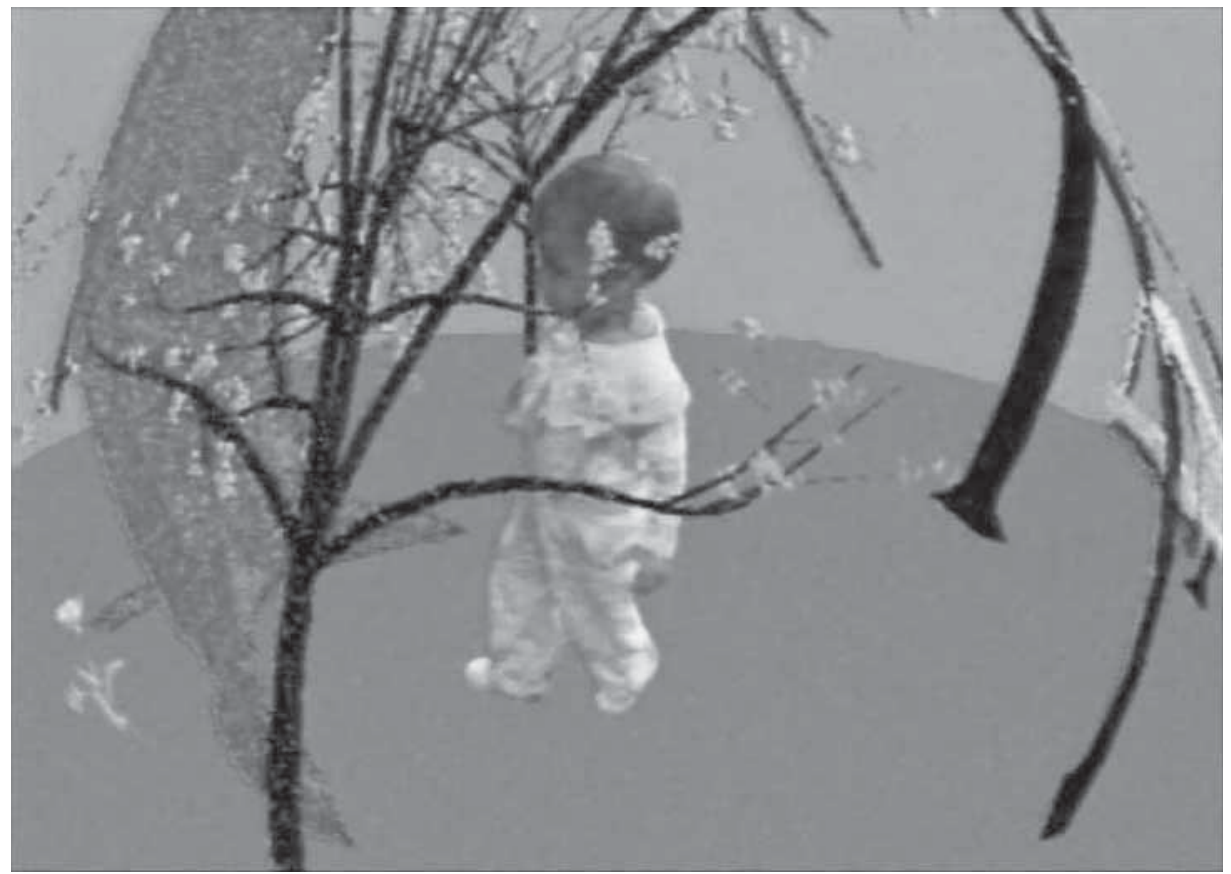

»The Garden, 21st Century Amateur Film«, ( ) T. Waliczky \& A. Szepesi, 1992, 1996. Mit freundlicher Genehmigung des Zentrums für Kunst und Medientechnologie, Karlsruhe

Abbildung 1: Tamás Waliczky: Der Garten. Ein Amateurfilm des 21. Jahrhunderts, 1992, Computeranimation, 4'27", Stills

Im ersten Moment fällt gar nicht auf, dass Waliczky das >MuybridgeParadigma ${ }^{3}$ angewandt, d. h. den Kopf des gefilmten Kindes so platziert hat, dass er stets den Mittelpunkt bildet, nur dass es nicht diszipliniert eine bildparallele Lokomotion vollführt, sondern das Territorium frei sondiert. Das Mädchen figuriert als Zentrum der dreidimensional konzipierten Bildwelt (im gezeigten Bildausschnitt der Animation ist das Köpfchen mittig auf der Hori-

3 Mit dem >Muybridge-Paradigmaく sei hier die über eine Bilderserie hinweg konstant gehaltene Zentrierung einer sich fortbewegenden Figur bezeichnet. Die Besonderheit der Experimentalanordnung des Fotografen Eadweard Muybridge bestand darin, seine Versuchspersonen und -tiere kameratechnisch orthogonal zu ihrer Laufrichtung zu erfassen. Als Animation zusammengesetzt haben derlei Fotoserien zur Folge, dass die Figur am Rumpf an der Stelle zu verbleiben scheint, während der Boden darunter (und der Hintergrund) wie beim Laufband durchzieht. Dass sich Waliczky damit auseinandergesetzt hat, beweist die Animation Wheel (aus der Serie Human Motions / Computer Mobiles, 1986-1988), in der er eine männliche Gestalt aus einer von Muybridges Serien mit einem computergenerierten Laufrad kombinierte (vgl. Szepesi 2001, 116). 
zontalen, aber stets im oberen Drittel der Vertikalen). Dafür waren computerseitige Analysen zur Lokalisierung der Bewegung des Mädchens sowie teils Anpassungen nötig, denn das Kind sollte in der Animation eine konstante Größe beibehalten (vgl. für technische Details Waliczky 1995). Um nun eine mögliche >Sicht des Kindes zu suggerieren, betonte der Künstler, worauf die Tochter ihre Aufmerksamkeit richtet oder - technisch präziser - wozu sie in ein Nahverhältnis tritt. All das, dem

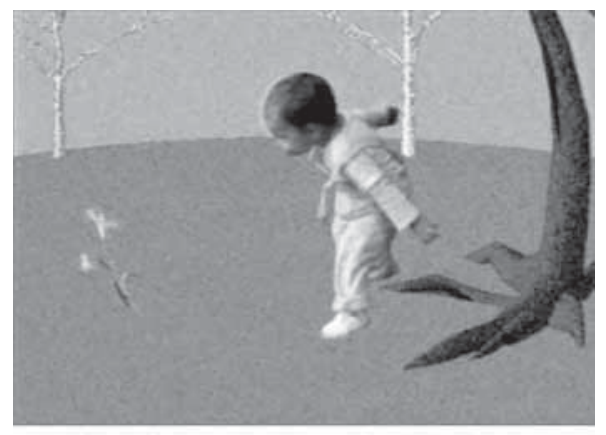
sie sich zuwendet, gewinnt an Prominenz, indem es sich optisch aufbläht und somit die Wahrnehmung dominiert. Alles vergrößert, weitet und dehnt sich in der Nähe des Körpers, während die Dinge mit zunehmender Entfernung schrumpfen (Abb. 2). »[N]ichts bewegt sich, aber alles wechselt die Form«, so bringt Lars Spuybroek das Faktum auf den Punkt, dass jede Regung der Protagonistin eine Änderung der Größenverhältnisse der gesamten kugelförmigen Welt nach sich zieht. Das Kind, so Spuybroek weiter, »ist zum Gravitationszentrum seines Feldes geworden, oder besser, einer Handlungssphäre - eines motorischen Feldes -, sein eigener Planet ... Dies ist nicht Perzeption, sondern Propriozeption. Alles wird unmittelbar innerhalb des Körpers vernetzt, wo das Gesehene auch das Berührte und Gefühlte ist, wo keine Unterscheidung mehr möglich ist, zwischen nah und fern, zwischen der manipulierenden Hand und der globalen Sphäre.« (Spuybroek 1997, 74). Diese auch als solipsistisch, egozentrisch oder traumartig beschriebene >Welt des Begehrens<

»The Garden, 21st Century Amateur Film «, C) T. Waliczky \& A. Szepesi, 1992, 1996. Mit freundlicher Genehmigung des Zentrums für Kunst und Medientechnologie, Karlsruhe

Abbildung 2: Tamás Waliczky: Der Garten. Ein Amateurfilm des 21. Jahrhunderts, 1992, Computeranimation, 4'27", Stills ist eine, in der sich Zuneigung wörtlich und grundsätzlich verlässlich als bilateral erweist und deren Begrenzung sich - fast behütend - über das Kind wölbt. 


\section{Perspektivierung}

Über die Verzerrung entscheidet der Künstler jedoch nicht je nach Gutdünken situativ, sondern sie folgt einem Satz von Regeln, den Waliczky erfand und WARP (WAterdRoP)-Perspektivsystem nannte. Um die Deformation zu automatisieren, programmierte Imre Kováts eine Software, die jede beliebige modellierte Computerszenerie gemäß den vorgesehenen Prinzipien krümmt. Man speiste das Gartenmodell sowie die $x-$-, $y$-, $\mathrm{z}$-Werte des Kopfes für jedes einzelne Bild in das Programm ein, um die Kopfkoordinaten des ersten Bildes festzustellen. Sodann justierte das Programm ausgehend von diesem Zentrum jeden Punkt des Gartenmodells. Diese Neuberechnung bildete gemeinsam mit der nächsten Positionierung der Protagonistin den Ausgangspunkt für das folgende Frame. Auch wenn die Wassertropfenperspektive im Prinzip alle Elemente der präsentierten Szenerie betrifft, lassen sich auch hier Stellen finden, wo die Perspektive dem Ausdruck offensichtlich zuwiderlief, sodass der Künstler davon absah, diese stur anzuwenden. So erlaubt die Umklammerung des Pfahles dessen Anschwellen nicht (Abb. 3). An anderen Stellen konnte der Bruch geschickt kaschiert werden: Beim Aufstieg an der Rutsche fällt nicht weiter auf, dass die rechte Hand des Kindes sich in der Luft abstützt, weil infolge der Verbreiterung der Treppe die Handführung außer Reichweite gerückt ist (Abb. 4).
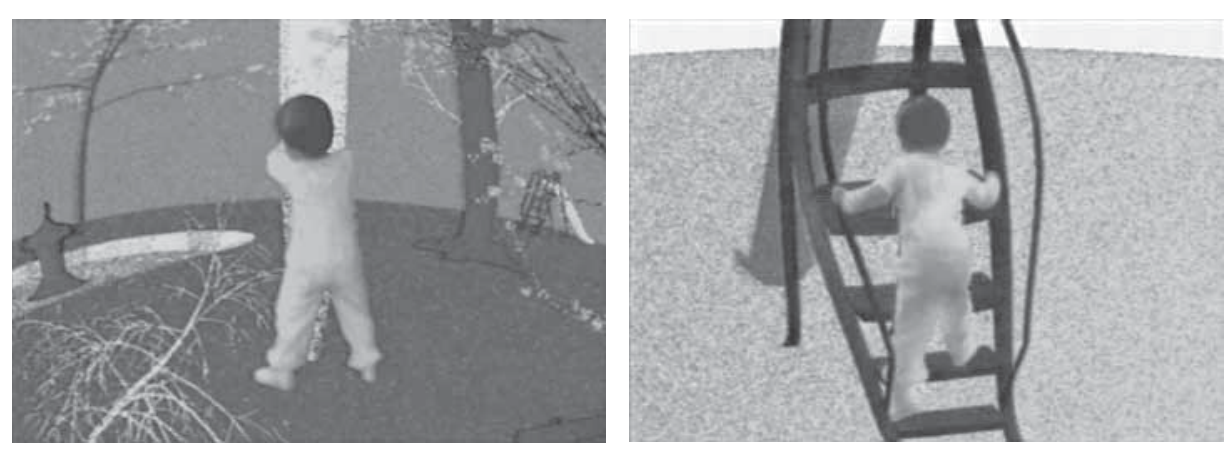

»The Garden, 21st Century Amateur Film«, (C) T. Waliczky \& A. Szepesi, 1992, 1996. Mit freundlicher Genehmigung des Zentrums für Kunst und Medientechnologie, Karlsruhe

Abbildung 3 und 4: Tamás Waliczky: Der Garten. Ein Amateurfilm des 21. Jahrhunderts, 1992, Computeranimation, 4'27", Stills

Waliczky verdeckte nicht, dass er seine Gartenmodelle mit flachen Bildern erstellte und in einem der letzten Arbeitsschritte beidseitig über ein spezielles Texturabbildungssystem kolorierte. Eine weitere Software ermöglichte es, sie in Hinter- und Vordergrund aufzuspalten, um die Modellhälften zum Schluss mit der Silhouette des Kindes in deren Mitte zu einer einzigen Ansicht zusammenzuführen. 
»Denken wir uns in den Raum hinein, in dem sich ein Kleinkind wahrnehmend bewegt: Es sieht alle Gegenstände in Beziehung zu ihm und seinen eigenen Bewegungen, denn es ist in seiner Welt der Mittelpunkt. Die konstruktive Entsprechung eines solchen >Wahrnehmungsraumes< ist eine Kugel, unabhängig davon, ob dies der >Wirklichkeit` der Raumverhältnisse entspricht. Denn wollen wir sehen und erleben, wie ein Kleinkind die Welt empfindet, müssen wir uns auf seine Weltkonstruktion einlassen und sie uns snachbilden`, damit wir sie überhaupt verstehen können." (Hünnekens 2007, 132-133). Zu behaupten, die Wassertropfenperspektive spiegle die Sicht eines Kindes oder gar dessen Empfindung wider, ginge wohl etwas zu weit. Denn erstens scheint dem Betrachter nicht aus >erster Perspektive< gezeigt zu werden, was das Kind sieht. Man vergegenwärtige sich hierfür Egoshooter-Spiele, die häufig die Option bieten, zwischen einer 1st- und einer 3rd-person-view zu wählen. Die Animation zeigt eher letztere Variante. Erst vor diesem Hintergrund einer nicht zur Verfügung stehenden expliziten Interaktion wird Waliczkys Aussage verständlich, seine Arbeit könne als eine Art virtuelle Realität gesehen werden, wobei man aber die virtuelle Welt eines anderen sehe. Nicht der Betrachter selbst entscheide, wohin zu schauen und was anzufassen ist, und dies verlange ihm Toleranz ab (vgl. Waliczky o. J.). In eine ähnliche Richtung argumentiert Margaret Morse (1997, 28), wenn sie schreibt: »Diese Arbeit stellt den Betrachter vor die schwierige Aufgabe, einen dezentrierten Standpunkt einzunehmen.« Erst mit neun Jahren erwirbt man überhaupt die Fähigkeit, eine nicht-egozentrische Perspektive imaginär einzunehmen (vgl. Hock 1984). Aber das Problem ist - zweitens - viel fundamentaler anzusiedeln, denn das Kind, das der gegebenen Perspektive entspräche, würde ständig zugleich nach oben, unten, vorn und zurückblicken. Um dies zu begreifen, bedarf die von Waliczky ausgeknobelte Perspektivierung einer näheren Erläuterung.

Im Unterschied etwa zu einer Fischaugen-Perspektive, die das Prinzip des Weitwinkelobjektivs $\left(180^{\circ}\right)$ ausreizt, handelt es sich bei der Wassertropfenperspektive um eine Ansicht, die die Dinge rund um eine Position herum, sphärisch $\left(360^{\circ}\right)$, zeigt. »Sie könnten nun sagen: `Natürlich sehe ich auch den vorderen Baum [jener im Rücken des Kindes; I. H. (vgl. Abb. 5)], da das Bild mit einer Kamera von außerhalb des Gartens aufgenommen wurde.< Aber das ist nicht wahr. Dieses Bild wurde aus dem Blickwinkel des Kindes gemacht.« (Waliczky 1995, 360). Daraus zu schließen, man könne die Betrachterperspektive mit der des Kleinkindes identifizieren, was - wie oben angedeutet - einer Deterritorialisierung des gewohnten eigenen Blickpunktes gleichkäme (vgl. Hansen 2004, 108-109), lässt eine wichtige Unterscheidung außer Acht. Waliczky $(1995,360)$ erklärt dies wie folgt: »Sie können sich diese Situation verdeutlichen, wenn Sie sich eine Person mit unendlich vielen Augen rund um den Kopf herum vorstellen. Diese Person könnte auch die Objekte wahrnehmen, die sich hinter ihr befinden.« Der Grund, warum uns diese Welt quasi 

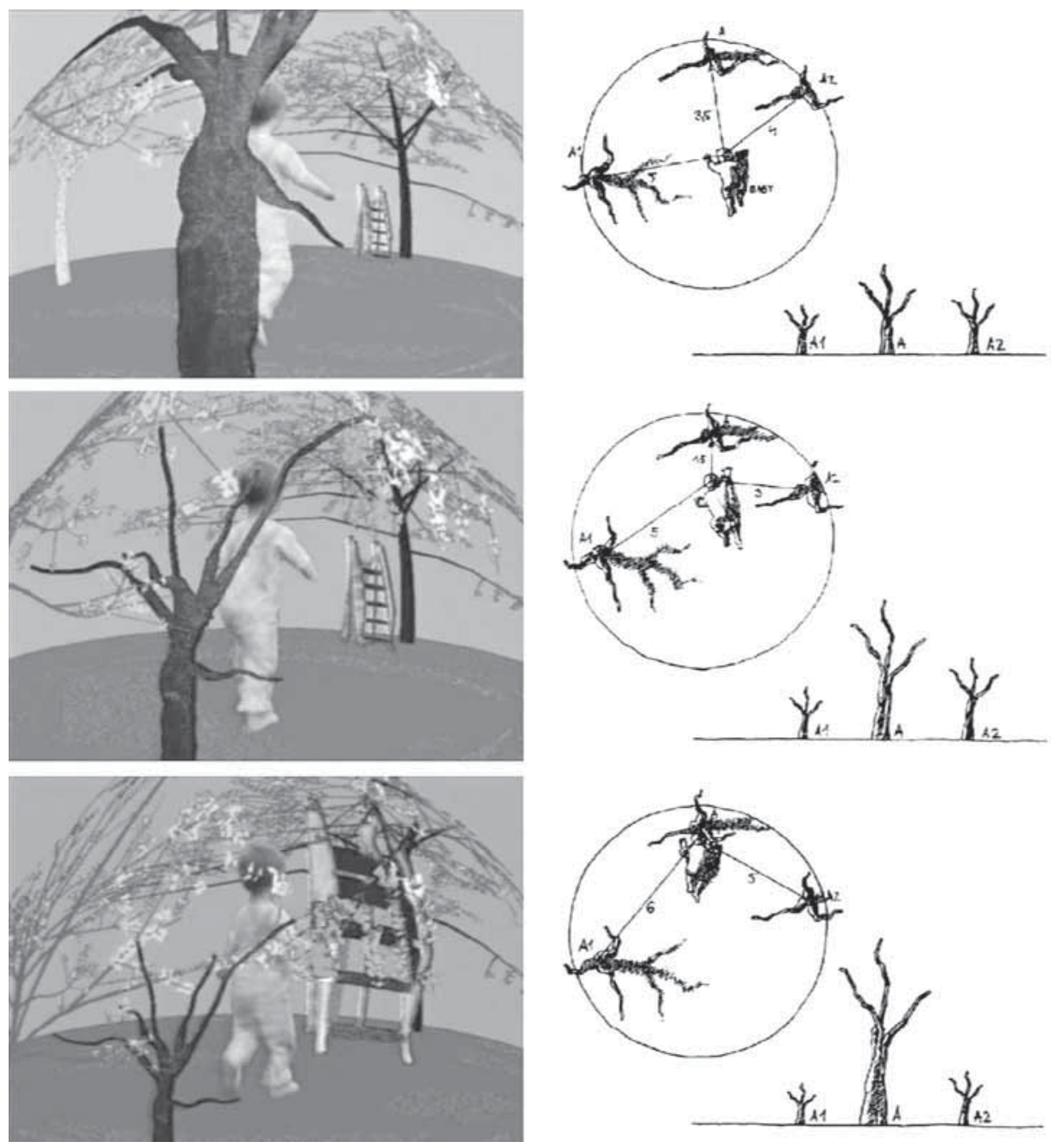

»The Garden, 21st Century Amateur Film«, ( ) T. Waliczky \& A. Szepesi, 1992, 1996. Mit freundlicher Genehmigung des Zentrums für Kunst und Medientechnologie, Karlsruhe

Abbildung 5: Tamás Waliczky: Der Garten. Ein Amateurfilm des 21. Jahrhunderts, 1992, Computeranimation, 4'27", Stills

überblicksmäßig von außen präsentiert erscheint, liegt in unserer Unfähigkeit, diese Sicht zu verkörpern. Selbst wenn wir die Animation panoramatisch in einem $\mathrm{CAVE}^{4}$ projiziert bekämen, brächten wir für die adäquate Rezeption der wassertropfenperspektivisch gezeigten Welt nicht die physiologischen

4 Der Fachbegriff CAVE ist die Abkürzung für >Cave Automatic Virtual Environment und bezeichnet eine immersive Vorrichtung, innerhalb derer man eine computergenerierte virtuelle Szenerie interaktiv erkunden kann. 
Voraussetzungen mit. Das Kind ist ein kleiner vieläugiger Alien, wenn wir unterstellen, dass es so sieht. ${ }^{5}$

\section{Sphärischer Horizont und Beobachtung zweiter Ordnung}

Dadurch dass die im Kopf des Kindes anzunehmenden, unendlich vielen Augpunkte beim Bildsujet verankert sind, wandern sie und dynamisieren den Raum. Im Wassertropfenperspektivsystem hat das Augpunkt-Bündel ebenso zahllose Fluchtpunkte darum herum als Pendants. Infolgedessen erhält auch der Horizont - in seiner Funktion und Bedeutung gleichbleibend - ein anderes Aussehen. Ist er traditionell als Gerade auf der Bildfläche verzeichnet, müsste er in Waliczkys System einen Kreis beschreiben (vgl. Abb. 6) bzw. eine rundliche Kuppel oder Blase um das Kind (vgl. Waliczky 1995, 359-361; Kluszczyński 2003). Obwohl das Setting mit dem sphärischen Horizont ein geschlossenes komplettes Universum vermuten lässt, wird dennoch nicht immer der ganze Garten gezeigt. »Theoretisch wäre es möglich gewesen, ein großes Gartenmodell mit allen Pflanzen und Objekten zu bauen und dieses Modell für den ganzen Film zu verwenden. Aber praktisch hätte dies sehr viel Zeit gekostet, deshalb zog ich es vor, mehrere, relativ kleine Modelle zu bauen, die nur die für die jeweilige Szene notwendigen Details des Gartens zeigten.« (Waliczky 1995, 356). Die Änderung des Sphäreninhalts nach jedem sSchnitt< demonstriert, dass es ein `Off $<$ jenseits des momentanen Horizonts gibt. Interessant ist nun, dass diese Grenze bzw. Portionierung über die ursprünglichen Filmaufnahmen beeinflusst, aber nicht determiniert sind: Der Künstler orientierte sich an den Elementen einer jeden aufgenommenen Sequenz, entschied schließlich aber frei, welche Objekte als Computermodell nachgebaut werden sollten.

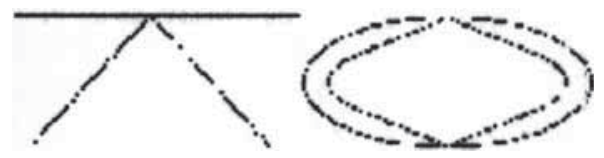

Aus: Waliczky 1995, S. 361, Abb. 11

Abbildung 6: Tamás Waliczky: Skizze des Horizonts der Wassertropfenperspektive, 1992, Computerzeichnung
Die Wahrnehmung des sphärischen Horizonts erfordert eine Sinnesphysiologie, die die Menschen nicht aufweisen, sie können nicht nach allen Seiten gleichzeitig spähen, so der Künstler: »[A]ufgrund der Tatsache, daß Sie, der Betrachter, nur zwei Augen haben, brauchte ich etwas, um Ihnen die Welt, die eine Person mit unendlich vielen Augen sehen könnte, zu verdeutlichen. Deshalb habe ich noch eine zweite Kame-

5 Allerdings könnte man einwenden, dass die durch die Zentralperspektive vorgeschlagene Reduktion, den Betrachter zu einem räumlich präzise fixierten, stillgestellten, entkörperlichten Zyklopen zu stilisieren, auch befremden müsste. 
ra außerhalb der Welt aufgestellt. Diese Kamera repräsentiert den Betrachter. Diese Kamera sieht ohne perspektivische Verzerrung. Ich würde sagen, daß die zweite Kamera keine Persönlichkeit hat.« (Waliczky 1995, 360-361). Die Grenze der Welt verläuft zwischen dem Kind und dieser bildlich indirekt ebenso vorhandenen Betrachterfigur. Letztere befindet sich folglich außerhalb und ist - glaubt man dem Künstler - als Betrachter ohne Eigenschaften vorzustellen. Dennoch wurde er mit einem eigens auf ihn zugeschnittenen $\mathrm{Zu}$ blick bedacht. Laut Niklas Luhmann (2008, 253) gehe es in der Kunst »schon lange um ein Arrangieren des Beobachtens von Beobachtungen, und nur dafür ist die Rollenasymmetrie, die Differenz der Beteiligtenperspektiven von Bedeutung. In jedem Falle muß der Künstler wie der Betrachter eine Beobachtung zweiter Ordnung vollziehen.«

Sehr richtig vermerkt Anne-Marie Duguet (1996), dass in dieser Arbeit selbst (abgesehen von einem effektiven Rezipienten, der noch dazukäme) zwei Weisen der Perzeption vorgeführt werden. Während der Beobachter, dem die Kamera svon außen< entspricht, lediglich über den Visus teilnimmt, agiert das Mädchen aktiv mit dem ganzen Körper. Duguets Intuition, es könne zu einer Überlappung beider Perspektiven kommen, ist zuzustimmen. Mögen sie auch meistens voneinander separierbar sein, eine Amalgamierung offenbart sich, sobald sich das Kind die Augen reibt (Abb. 7). Dann nämlich blendet die Umgebung ins Weiß. Nur ganz leicht angedeutet sind noch die Konturen der Außenwelt wahrzunehmen, fast als wären dies die Nachbilder auf der Retina des Kindes. Die Verschränkung ist dadurch gegeben, dass es also auch für den Beobachter zweiter Ordnung kaum mehr etwas
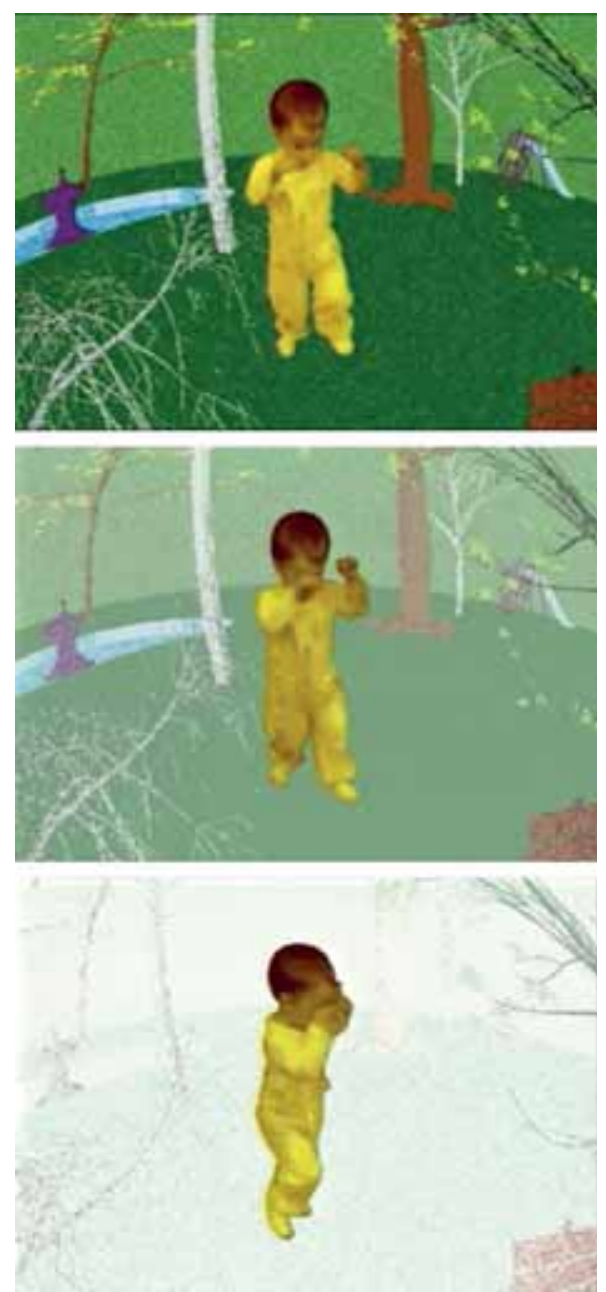

"The Garden, 21st Century Amateur Film«, (C) T. Waliczky \& A. Szepesi, 1992, 1996. Mit freundlicher Genehmigung des Zentrums für Kunst und Medientechnologie, Karlsruhe

Abbildung 7: Tamás Waliczky: Der Garten. Ein Amateurfilm des 21. Jahrhunderts, 1992, Computeranimation, 4'27", Stills und Skizzen 
zu erkennen gibt, wenn die Augen des Kindes getrübt oder geschlossen sind. Ein zweites Moment einer Überblendung lässt sich anführen, das diesmal den sexternen Zuschauer stärkt: Unschwer kann man einen Bruch innerhalb der Darstellung an der Stelle erkennen, wo das Kind eingefügt ist. Denn der Bildlogik zufolge nähme sich das Mädchen nur von einer Seite, teilweise gar als Rückenfigur wahr, anstatt dass auch hier gelten muss, dass es sich selbst wie alles andere auch - rundum sieht und nur dort eine Lücke entstünde, wo die Augen einen blinden Fleck schaffen: beim Kopf. Blinde Flecken sichtbar zu machen, ist gerade der Vorzug einer Beobachtung zweiter Ordnung. Damit ist also auch der Beobachter indirekt in die Szenerie integriert.

\section{Von der Darstellung zur Evokation von Welt}

In Der Garten sehen wir, wie ein Kleinkind sich seine Umwelt aneignet, indem es Entfernungen zurücklegt, Höhen bewältigt (erfolgreich: Leitersprossen, oder weniger erfolgreich: Baumstamm), Dinge festhält (erfolgreich: Pfosten, oder weniger erfolgreich: Libelle), etc. Gelingt nun das, was das Mädchen in seiner Welt tut, auch durch ein Bild? Die Animation visualisiert das tätige Dasein des Mädchens und zeigt, dass seine Handlungen auch erst hervorbringen, was die Qualität des Raums für das Kind ausmacht. Wenn wir nun den Betrachter an die Stelle des Mädchens, aber vor ein Bild setzen: Kann es auch da gelingen, sich zu einem Bild immer wieder anders zu positionieren und also so etwas wie eine Welthaftigkeit zu evozieren? Was in Der Garten Thema der Darstellung - Weltgestaltung durch aktives Zutun - ist, wäre dann in der Rezeption selbst zu erbringen.

Einen Weg ebnet uns Luhmann, wenn er sowohl Handeln als auch Erleben als (formdirigiertes) >Beobachten $<$ begreift. Beide Male geht es um das Markieren von etwas im Unterschied zu etwas anderem mithilfe von Formen (vgl. Luhmann 2008, 252). Dabei ist >Form< laut Luhmann eine Differenzfigur, »eine Unterscheidung durch eine Gestalt« $(1997,186)$.

Die nächste zu besprechende Arbeit stammt vom Schweizer Künstler Roman Signer, der - wie Waliczky - vornehmlich mit dem Thema 'Zeit in Verbindung gebracht wird. In seiner Videoarbeit Heufieber (2006) zielt er jedoch primär auf die Konstitution von >Raum<. Analoges gilt für Der Garten. ${ }^{6}$ Das Ungewöhnliche an Signers Arbeit besteht darin, dass - um vorerst noch in Luhmanns Terminologie zu bleiben - für den Nachvollzug bzw. die Ergründung der Formentscheidung des Künstlers in der Rezeption nacheinander verschiedene Formen herangezogen werden (müssen). Bei Signer sind diese differenzierenden Formen als Bewegungen gepaart mit Erfahrungswissen zu

6 So bemerkt Sven Drühl $(2000,140)$ richtig, in Der Garten »steht nicht etwa der Begriff der Zeit im Zentrum des Interesses, um das Konzept des Films zu überwinden bzw. zu erweitern, sondern vielmehr der Raum.« 
verstehen und zielen in Luhmanns Ausdrucksweise am ehesten auf >Stellen (Raum). ${ }^{7}$ Diese erfahrungsbezogenen Formen korrespondieren mit je einem qualitativen Raumempfinden. Wenn ein Bild den Betrachter normalerweise in ein bestimmtes Verhältnis zu sich situiert - man denke an den einen einzigen Augpunkt bei einem zentralperspektivisch konstruierten Bild -, so sickern hier über die Wahrnehmung dieses speziellen Videobildes nacheinander Facetten ins Bewusstsein, die bewirken, dass man sich nicht nur auf eine Weise zum ikonischen Bildraum verhält. Oder nochmals anders ausgedrückt: Der Künstler komponiert die Szenerie so geschickt, dass der Rezipient darin zeitlich sukzessiv und den Raum betreffend mehrmals Unterscheidungen vornimmt.

\section{Eine surreale Szene}

Hier greifen wir nun aber zu weit vor, zunächst folgt eine Beschreibung des Videos Heufieber. Es handelt sich um eine unprätentiöse Aufzeichnung eines in einem Innenraum inszenierten Vorgangs, wobei die Kameraeinstellung durchweg unverändert blieb: kein Schnitt, kein Schwenk, kein Zoom (Abb. 8). Zunächst hat der Betrachter genügend Zeit, sich mit dem Exposé vertraut zu machen. Man sieht ein betont überschaubares, beinahe leeres Rauminneres: den Boden, die Decke und drei Wände des Atelierraums in St. Gallen. Da man anhand der Kantenlinien dieses Kubus problemlos den Fluchtpunkt ermitteln und eine betont zentralperspektivische Anlage feststellen kann, symbolisiert dieses Dispositiv
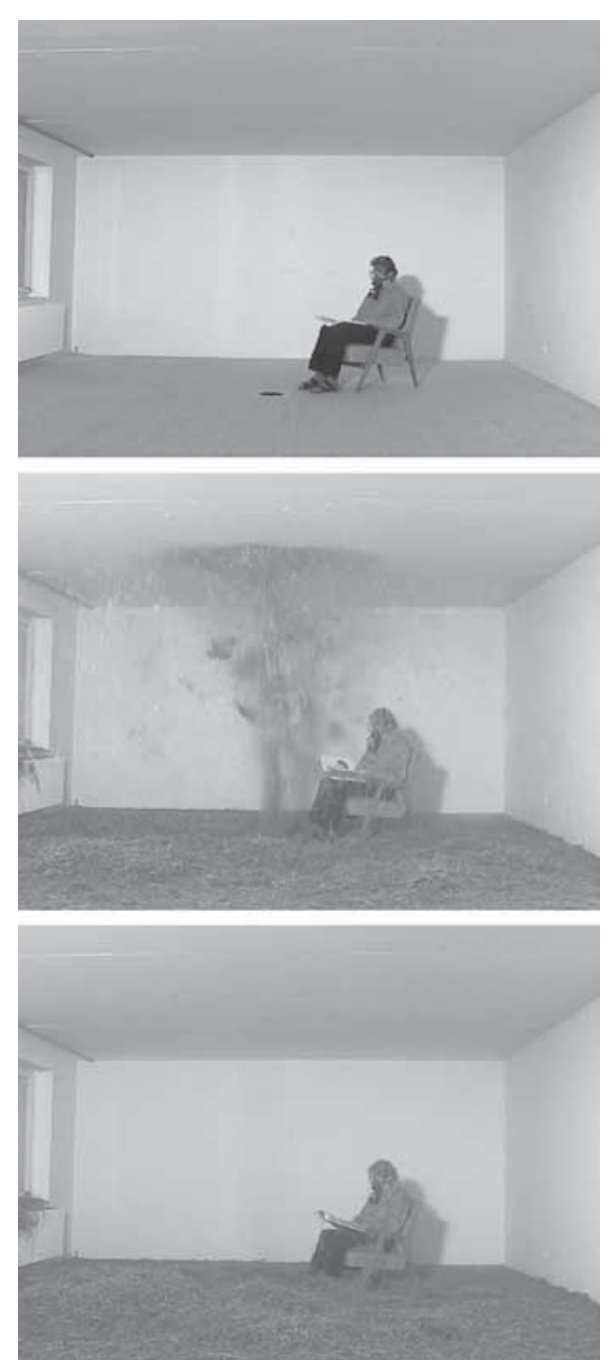

Roman Signer, Heufieber 2006, Video Aleksandra Signer

Abbildung 8: Roman Signer: Heufieber, 2006, Video, 2'30", Stills 
ein apriorisches Raumgefüge, in das alles Vorkommende nachträglich integriert wird. Eine Figur ist auch vorhanden: Der Künstler hat mit Atemmaske und Schutzbrille auf einem Sessel Platz genommen. Ein Bilderbuch liegt aufgeschlagen auf seinen Oberschenkeln. Nahe seinen Füßen, die mit Socken und Sandalen bekleidet sind, bemerkt man einen schwarzen runden Fleck, der sich bald als Loch im Holzboden erweist. Durch diese Öffnung wird Heu in den Raum gepumpt. Mehr passiert während des ganzen Videos nicht: Signer lässt sich vom Maschinendröhnen und vom getrockneten Gras, das mit Wucht vor ihm an die Decke schießt und sich sodann rund um ihn und auf ihm verteilt, in seiner Tätigkeit als Bilderbuchblätterer nicht im Geringsten stören. Ein absurder Vorgang wird nüchtern geschildert und vom Betroffenen völlig gelassen hingenommen. Nach knapp zweieinhalb Minuten hört der Heufluss auf. Zum Schluss wird noch ein Lüftchen mit feinem Staub in den Raum gehaucht. Der Künstler bleibt sitzen.

Aber am Ende hat sich doch viel mehr ereignet als die offensichtliche videotechnisch aufgezeichnete Begebenheit. Der zunächst so unzweideutige Raum verändert sich im Verlauf des Vorgangs. Zur figurierten Bewegung treten quasi en passant noch weitere hinzu, die den gesamten Raum umkrempeln. Neben dem surrealen, aber letztlich auch gleichförmigen Geschehen kippt die Ansicht mehrmals durch punktuell einsetzende >Mechanismen<, die man nicht unbedingt sieht, aber dennoch wahrnimmt. Was ist damit gemeint?

\section{Erlebnis-Raum und Äonszeit}

Es bleibt frappierend, dass ein faktischer Ablauf, der durchaus unterschiedliche zeitliche Dichten aufweisen kann, eine sich darin ereignende andersartige Transienz erzeugt. Im Unterschied zu einer messbaren Taktung fasst der französische Philosoph Henri Bergson die Zeit qualitativ, was er am Beispiel des Sich-Dehnens der Zeit erklärt. Hierfür stellt er nicht etwa ein abstraktes Konzept vor, sondern eine lebensweltliche Situation mit einem konkreten Verlauf: »Will ich mir ein Glas Zuckerwasser bereiten, so muß ich, was immer ich anstelle, das Schmelzen des Zuckers abwarten. Diese geringfügige Tatsache ist voll von Aufschlüssen. Denn die Zeit, die ich warten muß, ist nicht mehr jene mathematische [...]. Sie fällt zusammen mit meiner Ungeduld, d. h. mit einem Teil meiner eignen Dauer, der weder willkürlich ausdehnbar noch abkürzbar ist. Nicht mehr Gedachtes ist hier, sondern Gelebtes « (Bergson 1907/1912, 16). ${ }^{8}$ Für diese Erlebens-Zeit stand in früheren Epochen ein Name, den Paul Good in Erinnerung ruft: »Heraklit hat dafür bereits um 480 v. Chr. auch einen Gott geliefert, indem er den Aion (den Äon, die Welt- und Erlebbegriffs (vgl. Good 2002; vgl. ferner als Antwort dazu: Withers 2007). 
niszeit) ein spielendes Kind nannte, das nach Herzenslust auf- und abbaut, formt und zerstört. Äonszeit zirkuliert auf der Oberfläche der Chronoszeit. Sie wird im Spiel, im Leben, in der Wahrnehmung der Dinge, bei den Gegebenheiten des Bewusstseins überall erfahren." (Good 2009, 82-83).

Wenn man nun versucht, das was für die Zeit bereits prominent ausgeführt wurde, auf den Raum zu beziehen, so würde man in Analogie zu Bergson vom kartesischen Apriori absehen und den lebensweltlichen Erfahrungsschatz aktivieren, der einem bestimmte Situationen auf spezifische Weise erscheinen lässt.

Unter diesen Vorzeichen lassen sich die Eindrücke beschreiben, die Signers Arbeit hinterlässt: Die vertikal in die Höhe gerichtete Bewegung kommt unerwartet. Sie kennen wir fast nur von Wasserfontänen (vgl. Abb. 9). Durch die Decke erfährt der Heustrahl jedoch eine abrupte obere Begrenzung, womit
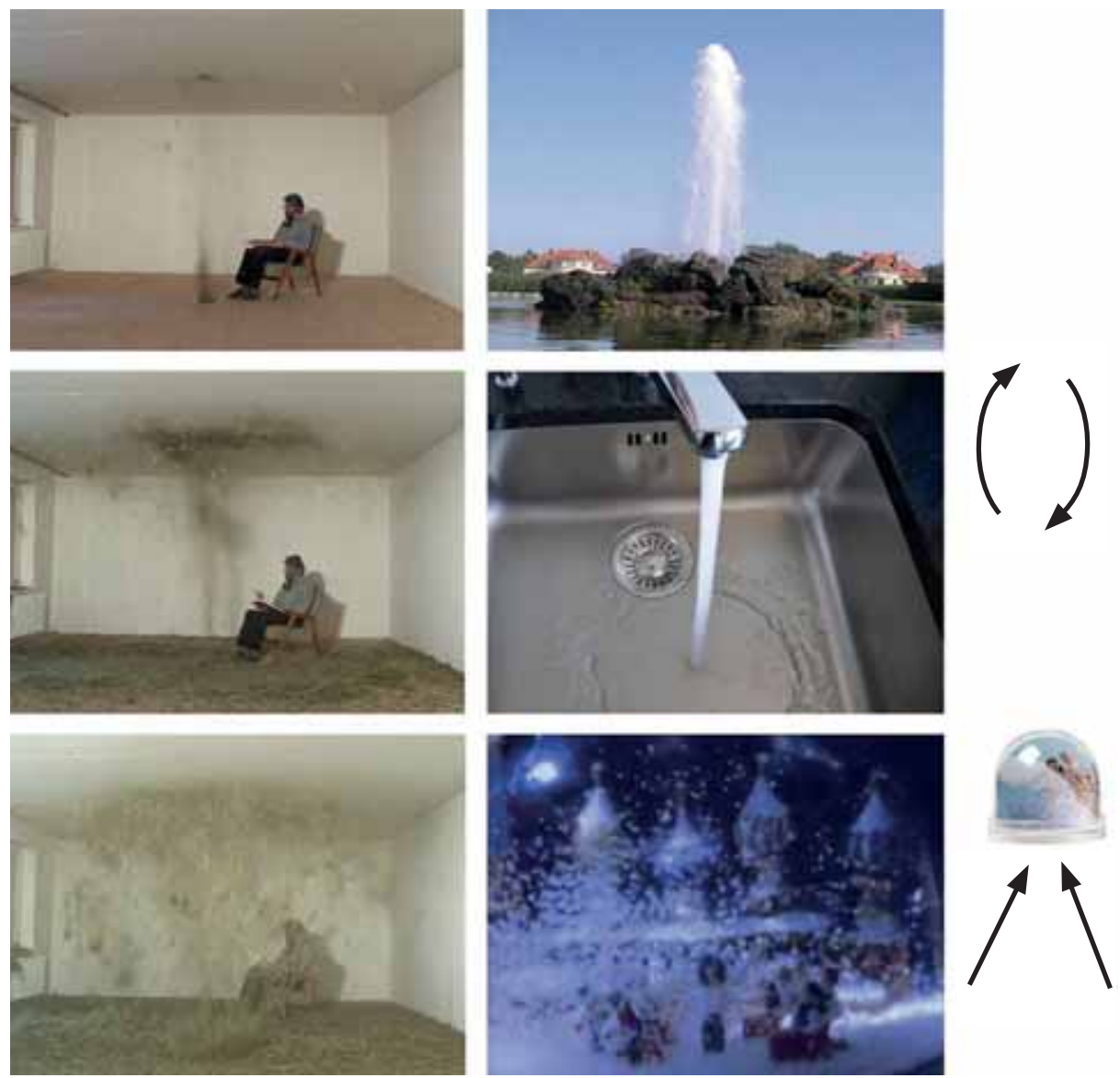

Roman Signer, Heufieber 2006, Video Aleksandra Signer

Abbildung 9: Roman Signer: Heufieber, 2006, Video, 2'30", Stills (linke Seite; rechte Seite: Vergleichsbilder) 
die Assoziation an eine Fontäne sogleich nachhaltig gestört wird. ${ }^{9}$ Die kaum separiert wahrnehmbare nächste Überraschung erwartet den schwerkraftgeprüften Betrachter, der sich vorgestellt hätte, dass das Heu nach dem Auftreffen an der Decke sogleich senkrecht kehrtmachen würde. Stattdessen unternimmt das mit Wucht hochgeschleuderte Heu an der Decke eine schier verblüffende horizontale Bewegungsfortsetzung radial in alle Richtungen. Es stiebt mit erstaunlicher Geschwindigkeit auseinander und fällt fernab der Heusäule in einzelnen Packen wieder auf den Boden. Der Auftreffpunkt an der Decke ist ein besonderer Ort, fast eine zweite Quelle, denn hier wird umverteilt, es entfaltet sich etwas. Trotz des dehydrierten Materials, mit dem Signer operiert, assoziiert man aufgrund der Art der Bewegung unwillkürlich einen Wasserfall oder einen Strahl ins Bassin und dreht damit den Raum in der Vorstellung kurzzeitig auf den Kopf.

Durch das Auseinanderstreben an der Auftrefffläche wird der Raum in seiner Ausdehnung ausgelotet. An der Decke scheinen sich wolkenartige Flecken zu bilden, die sich dann im Vordergrund als helle, schräg fallende Halme herausstellen. Beobachtet man die Halme in Ruhe, so nimmt man allmählich zur Kenntnis, dass hinter der aufnehmenden Kamera eine künstliche Lichtquelle angebracht sein muss, erkennbar auch an Signers Schattenwurf an der Wand. Die offensichtlich vorhandenen Scheinwerfer lassen die Strohhalme im Vordergrund hellgelb aufleuchten. Irgendwann bemerkt man ihre scheinbare Zickzackbewegung. Dadurch hat man den Eindruck, sie ändern die Richtung, weil sie an eine unsichtbare Wand stoßen (de facto freilich drehen sich die gebogenen Halme nur um die eigene Achse). Unversehens also hat sich scheinbar eine weitere Wand zwischen dem Geschehen beim Heu und dem Betrachter eingezogen. Die Grashalme sind durch die Beleuchtung auffällig genug, um eine Frontfläche zu bilden, ohne den Durchblick zu verhindern. Damit rückt das Geschehen der Heufontäne plötzlich vom Betrachter ab. Während man sich als Zuschauer kurz davor noch im selben Raum verortet hatte, sieht man sich nun durch die vermutete vierte Wand von der Kammer getrennt. Damit wird zugleich der Größenmaßstab hinterfragbar. Der nun vor Augen stehende Innenraum und dessen angenommene Ausmaße verlieren plötzlich ihre Selbstverständlichkeit. Schaut man in einen Guckkasten? Dass man diesen Gedanken hegen kann, verdankt sich dem gewählten Ausschnitt des Videos. Es kann hinsichtlich der Orientierung (Umkehrung) oder der Ausdehnung des Raums täuschen. Einzig der Sitzende, dessen Anwesenheit nun als Größenreferenz endlich eine Berechtigung erhält, gibt den Hinweis darauf, dass es sich wohl doch nicht um ein miniaturisiertes Raummodell oder gar um eine Art Schneekugel handelt. ${ }^{10}$ Mit letzterer gelänge es immerhin, die

9 Eine Ausnahme in Signers Euvre selbst bildet die Arbeit Fontana di Piaggio (1999).

10 Genau dieselben gestalterischen Mittel - durch einen immer stärker werdenden Schneefall im Vordergrund - wandte Michael Jackson im Musikvideo Black or White (1991) an, um die Überleitung von einer Tanzszene zu einer Schneekugel mit entsprechenden Plastikfigür- 
sonst seltene Erfahrung eines Niederschlags in einem geschlossenen Inneren zu verbinden.

Am Ende, als kein Heu mehr aus dem Boden schießt und der Maschinenlärm versiegt, lichtet sich die Sicht langsam und man gehört als Betrachter nach und nach immer mehr auch selbst wieder diesem Raum an. Es ist nun aber ein Raum, der einige Volten hinter sich hat: Zunächst war es einer der entweder partiell die Schwerkraft überwunden hat oder vermeintlich auf den Kopf stand; dann einer, der geschrumpft ist bzw. den Betrachter mittels einer eingezogenen Wand distanziert und vor dem Staub geschützt hat.

\section{Wahrnehmung mit dem Körper}

Wenn man das Euvre des Künstlers überblickt, so erkennt man, dass Signer es anfangs vermied, in den Videodokumentationen zu seinen Aktionen selbst in Erscheinung zu treten. Seit den späten 1970er Jahren jedoch tauchen zunächst ein Arm oder ein Bein, später dann der ganze Signer auf. Er sagte von sich selbst: »[M]eine Auftritte als Körper kamen langsam, und sie sind auch sehr kurz.« (zit. n. Van den Bosch 2000, 10). War er oft nur der Initiator eines Prozesses, damit beschäftigt zu tun, was gerade ansteht - diverse Zündungen, Würfe, etc. -, verblieb der Experimentator zunehmend im Bildausschnitt, da er die von ihm inszenierten Kräfte am eigenen Leib so nah wie möglich erleben wollte: »Ich bin derjenige, der ertragen muss, was er angezettelt hat, der da durch muss.« (zit. n. Van den Bosch 2006, 30, übers. v. I.H.). Dabei benötigt Signer nicht selten Sturzhelme, feuerfeste Schutzanzüge und Handschuhe.

Für die Vermittlung seiner Aktionen ist Signer auf die Film- oder Videodokumentationen angewiesen. »[D]ies sind Arbeiten, die gemacht wurden, um gefilmt zu werden, und sie sollen als Projektionen im Raum serfahren den. Wir als Betrachter wollen [...] rauthentische< Erfahrung, werden sie aber nie bekommen. Gibt uns Signer diese Erfahrung?«, fragt Brian Butler (2008, 18). Mit Heufieber beschert uns Signer sicher nicht die Erfahrung, die er selbst macht. Nur welche wäre dies überhaupt? Wenn er sich trotz aller Gefahr mitten ins Geschehen begab, ging es ihm meistens darum, einen anders nicht zu gewinnenden Sinneseindruck zu erheischen. Umso mehr erstaunt Signers ungewöhnliche Teilnahmslosigkeit oder Ignoranz gegenüber dem Geschehen in Heufieber. Anstelle einer konzentrierten Fokussierung vertieft er sich in das Buch. Dies wirft etliche Fragen auf: Ist Signer in seiner Beweglichkeit eingeschränkt und betrachtet er deswegen die Außenwelt nur vermittelt über den Bildband? Anklänge an das Höhlengleichnis und die Thematisierung des

chen zu bewerkstelligen (vgl.: aus dem Video stammt auch das Vergleichsbild in Abb. 9; vgl. Beier / Neely 1992). Betrachtet man Signers Euvre, wäre es darüber hinaus durchaus denkbar, dass es sich um einen nachgebauten verkleinerten Innenraum handeln könnte, wie es beispielsweise bei Hauptstraße und Nebenstraßen (1998) oder Hedendaagse (1982) der Fall ist. 
Horizonts sind erkennbar. Die typischere Haltung für ein projektionsbasiertes Fenster nach draußen wäre die Fernsehkultur. Aber das hätte potenziell die Konnotation, dass der Fernsehende so absorbiert ist, dass er nicht einmal mehr merkt, dass im eigenen Haus ein Sturm tobt. Hingegen passiert dies beim Bilderbuchblättern nicht, zu sehr ist er durch das auf die horizontale Unterlage fallende Heu gestört. Das muss er angestrengt ignorieren.

Diese Assoziationen dürfen nicht darüber hinwegtäuschen, dass die Situation für Signer als Allergiker existenziell ist, ähnlich wie in seiner Arbeit Bett (1996), wo ein Modellhelikopter gefahrvoll knapp über den auf einer Liegestatt vermummten Künstler kreist. ${ }^{11}$

Eine derartige Bedrohung wird dem Betrachter nicht aufgebürdet. Aber eine sauthentische< Erfahrung ist mit Heufieber sehr wohl zu erhalten, d. h. eine, die nicht im Video konservierbar ist, sondern von dort aus nur getriggert werden kann, zur Instantaneisierung im jeweiligen Hier und Jetzt. Genauso wie von einem Jetzt »nur mit Bezug auf ein Subjekt gesprochen werden [kann], das aktual etwas erfährt und dem eine bestimmte Perspektive eigen ist «, lässt sich laut Wolfgang Röd $(1991,55)$ auch der Raum subjektiv betrachten, sofern er »der Raum [ist], in dem wir leben. Als solcher hat er eine ausgezeichnete Stelle, auf die wir mit dem Ausdruck shier hinweisen und von der aus wir uns in bezug auf Dinge orientieren.« (Röd 1991, 60). In Signers Video braucht es also die Realisation durch den Rezipienten, dessen Erlebnis im Bildkonzept selbst antizipiert und einkalkuliert ist. Die Videoaufzeichnung ist nun nicht mehr lediglich ein dokumentarisches Hilfsmittel, sondern dient Signer als unabkömmliches Medium, das sich als konstitutiv erweist für die Möglichkeit, den Raum mehrfach zu reinterpretieren.

Mit seinem Diktum eines sSehens mit dem Körper knüpft Mark Hansen (2001) an Bergson an, für den sich die körperlichen Fakultäten Empfindung und Erinnerung sowohl bei der Selektion als auch beim Gesamteindruck der Wahrnehmung maßgeblich beteiligen. »Man muß aber bedenken, daß unser Körper kein mathematischer Punkt im Raume [Zentralperspektive!; I. H.] ist, daß seine virtuellen Handlungen sich mit den aktuellen vermengen und durchdringen, mit anderen Worten, daß es keine Wahrnehmung ohne Empfindung gibt. Die Empfindung ist demnach das, was wir vom Innern unsers Körpers dem Bilde der äußeren Körper zufügen« (Bergson 1991 [1896], 45). Mit Bergson, könnte man nun sagen, weiß der Rezipient um die lange Weile der Zuckerschmelze wie auch um einen mit einem Fontänen-, Wasserfalloder Puppenhausschema konnotierten Raum, zu dem er sich über sein >Hier positioniert und wozu er in Korrelation für das Gesehene `Konsequenzen (Drehungen, Verkleinerungen) entwickelt. Auch andere Denker nach ihm

11 Marcel Zünd, Kurator der Ausstellung Heuen im Appenzellerland: Geschichte, Bauernarbeit, Kultur des Appenzeller Volkskundemuseums in Stein (29.4.-8.10.2006), für die Heufieber exklusiv entstand, danke ich für Hinweise zur Entstehung der Arbeit (persönliche E-MailKorrespondenz, 18.4.2012). 
postulieren, dass man einen Schritt über das eigentlich Vorliegende dazuinterpretiert. Für Brian Massumi $(1998,21)$ - ebenso unter Rekurs auf Bergson - hängt dies mit der erfahrungsgesättigten, synästhetischen und kinästhetischen Natur des Sehens zusammen. Er berichtet von einem Beispiel einer Kollegin: Im Anblick einer gewundenen Jugendstilranke sehen wir nicht eine Spirale, sondern die Bewegung: ein Spiralen. Susanne K. Langer »reminds us that we see things we don't actually see. We all know it, but we tend to brush it off by calling it an illusion, as if something is happening that isn't real and doesn't have anything important to say about experience. But isn't ssomething happening the very definition of real? [...] Instead of calling it an illusion this movement we can't actually see, but can't not see either - why not just call it abstract? Real and abstract. The reality of this abstraction doesn't replace what's actually there, it supplements it. We see it with and through the actual form. It takes off from the actual form. The actual form is like a launching pad for it. We wouldn't see the movement without the actual design being there, but if we only saw an actual design we wouldn't be seeing what it is we're seeing - a motif. The actual form and the abstract dynamic are two sides of the same experiential coin. They're inseparable, they're fused, like two dimensions of the same reality. We're seeing double.« (Massumi 2007, 72-73). Im Unterschied zum Beispiel, das hier Langer vor Augen steht, ist in Heufieber eine Art von Bewegung explizit vorgeführt. Die Analogie besteht aber darin, dass wir mit und über Motive (hinaus) noch etwas anderes wahrnehmen - und zugleich nicht wirklich sehen: hier Raumkonfigurationen jenseits des zentralperspektivischen Videobildes. Im Beispiel von Heufieber kommt freilich der verblüffendste Effekt noch dazu, sobald diese Raumkonfigurationen in der Wahrnehmung moduliert werden, was genauso Fragen der Wahrnehmung explizit in den Vordergrund treten lässt. Das >Fieber im Titel deutet vielleicht auch an, dass die Wahrnehmungsvarianz fiebrig-halluzinierend zunimmt. Dass es dem Künstler um solcherlei Perzeptionsphänomene geht, belegt ein kurzes Zitat auf dem Buchrücken der Publikation Visuelle Wahrnehmung, die Signer mit den Worten empfiehlt: »Dieses wunderbare Buch von Jürg Nänni ist für alle Augenmenschen eine Notwendigkeit, eine Quelle der Erkenntnis sowie ein Anreiz zu eigenem visuellen Experimentieren. Insbesondere Gestalter, Künstler, Grafiker, Mediziner, Biologen, Psychologen und Hirnforscher werden Freude haben an diesem Werk.« (Signer 2008, Buchrücken).

\section{Umschnappen im bewegten Kippbild}

Ist bei der Raummodulation von einer Alternierung von Wechsel und Dauer zu sprechen oder gilt, was Bergson als Paradox formulierte, nämlich dass das Veränderliche dauert? Dies »heißt einmal, es tritt fortgesetzt Anderes, Neues, Unerwartetes in Erscheinung, sodann aber auch, das Verschwindende, das 
Vergangene, hört nie auf, weiter zu wirken und anders das Gegenwärtige und das Zukünftige zu beeinflussen.« (Good 2009, 135). Trotz Bergsons Gedanke, dass Wechsel dauern, bleibt rätselhaft, wie ein an sich kontemplatives, relativ monotones Geschehen so vielfältige Sensationen bereithalten kann. Zugegeben, so ganz einförmig ist der Vorgang in Heufieber nicht, Signer produziert darin durchaus verschiedene Zeitqualitäten: eingangs das Warten, dann
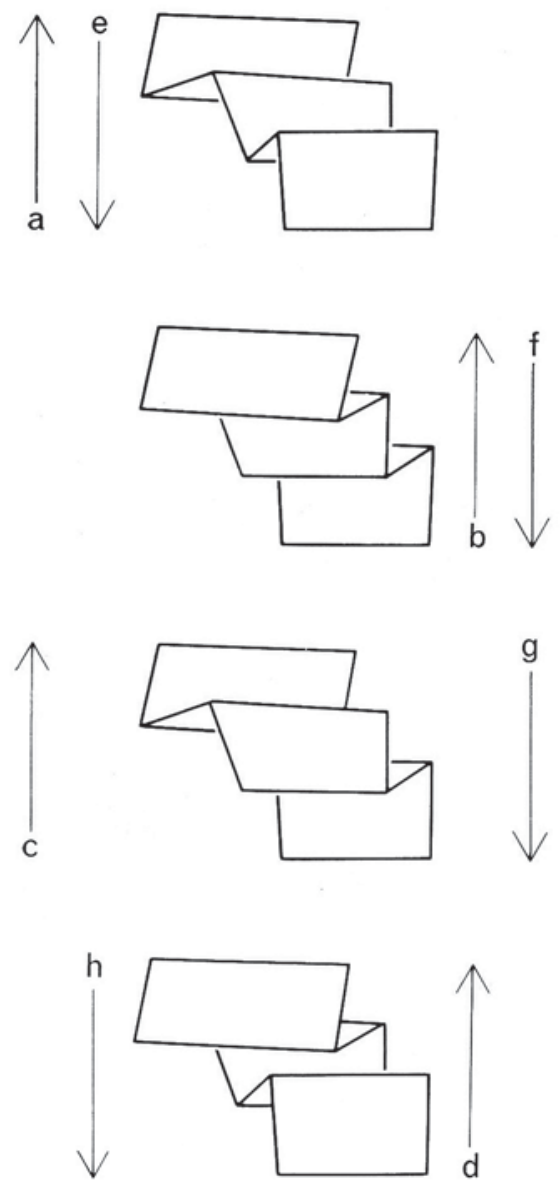

Aus: Bucher 1961, 12

Abbildung 10: Josef Albers, Analysezeichnung der grafischen Konstruktion des Glasbildes Stufen (1931), o. J. das sich durch einen Hauch von Rauch ankündigende Dräuen, daraufhin das eruptive Hochschnellen, das langsamere Herunterrieseln, das allmähliche Versiegen. Aus dem Nebeneinander und Nacheinander verschiedener Bewegungsarten wird die Aufmerksamkeit jeweils durch Unterschiedliches absorbiert, und damit werden Reinterpretationen - hier des Raums - erleichtert. Erst nachdem man sich an die nach oben und dann zur Seite gerichtete dominante Bewegung gewöhnt hat, widmet man sich auch der Peripherie, dem Heuregen. Und erst nachdem auch hier über viele redundante Einzelbeobachtungen eine gewisse Sättigung eingetreten ist, stellt sich die gesamte Szenerie plötzlich wieder unter anderen Vorzeichen dar. Somit bestätigt sich abermals, was Rudolf Arnheim $(1965,196)$ postulierte: »Das Phänomen ändert sich, je nachdem welcher Teil des Musters die Aufmerksamkeit des Betrachters auf sich zieht.«

Die vor sich gehenden Bildraumumschwünge in Signers Video könnte man analog $\mathrm{zu}$ den sspringenden Perspektiven in der Malerei denken. ${ }^{12}$ Als Beispiel dient eine Grafik, die wahrscheinlich Josef Albers

12 Mit dem folgenden Vergleich mit den weitläufig bekannten Kippbildern wird nicht angestrebt, die Arbeit Signers vollumfänglich oder in ihrer künstlerischen Qualität zu erfassen. Vielmehr geht es um das Verdeutlichen eines modus operandi, der in der Rezeption ein Raumkippen bewirkt. Im Falle von Zeichnungen ist dieser genuin bildliche >Mechanismus< als Vorgang einfacher zu begreifen als im Video, das auf einer anderen Ebene bereits zeitbasiert ist. 
selbst angefertigt bzw. kommentiert hat (Abb. 10). Es handelt sich um eine Bewegungsaufschlüsselung der kleineren Darstellung im Glasbild Stufen von 1931. Albers unterschied acht Leserichtungen, die er jeweils mit den Pfeilen markierte und die allesamt in der vieldeutigen Darstellung kondensiert sind. Seine geometrischen Liniengefüge lassen dreidimensionale Konstellationen erkennbar werden. Die oberste der vier treppenartigen Gebilde scheint man von unten zu betrachten, wobei man imaginieren kann, die Treppe hochzusteigen oder zu beobachten, wie jemand herabsteigt. Bei der zweiten Darstellung hingegen blickt man eher von schräg oben und könnte sich nicht mehr so einfach in die Lage versetzen, die Stufen selbst zu nehmen. Die beiden unteren Darstellungen liegen dazwischen, da sie in der Mitte ein zweifach lesbares Kippelement aufweisen. Mal scheint man hier eine Vogel- mal eine Froschperspektive einzunehmen, in Abhängigkeit davon, wo man die Betrachtung sbeginnt. Man sieht dasselbe gleichzeitig anders, und in diesem Fall resultieren daraus sich überlagernde Bewegungen, die zugleich unterschiedliche Betrachterstandpunkte implizieren. Im Zusammenhang mit dem beliebtesten aller Kippbilder - dem Hasen-Entenkopf - wurde diesbezüglich von einer »topologischen Doppelgesichtigkeit« (Heßler/Mersch 2009, 25) gesprochen, die laut Sybille Krämer zur Folge hat, dass der Betrachter jeweils auf unterschiedliche Blickachsen orientiert wird. Krämer (2009) rekurriert auf die weitreichend bekannten Überlegungen zu Kippeffekten durch Ludwig Wittgenstein, der dafür den Terminus sAspektwechsek in die Diskussion einbrachte. Das Denken tritt zur Wahrnehmung hinzu, wenn der Sinneseindruck mal als dieses und mal als jenes interpretiert wird, d.h., wenn man die Änderung wie eine Wahrnehmung beschreibt, "ganz als hätte sich der Gegenstand vor meinen Augen geändert« (Wittgenstein 1984c, § 476), wobei sich aber unbegreiflicherweise »nichts geändert hat, und sich doch Alles geändert hat.« (1984b, § 474). Für Wittgenstein zeigt sich »das Beteiligtsein des Denkens genau beim Wechsel des Aspektes, just in dem Augenblick, in dem sich das wirkliche Umkippen vollzieht, denn diesem Umschalten entspricht ja keinerlei Pendant in einem >Umkippen der Zeichnung selbst. Wittgenstein stellt fest: Wir können den Aspekt, nicht aber den Aspektwechsel sehen. Doch um diese Fähigkeit zum Aspektwechsel gleichwohl zu erfahren, muss ich mich - wie Wittgenstein betont - >mit dem Objekt beschäftigen ... insofern ist das Erleben des Aspektwechsels auch gleich einem Tun.« (Krämer 2009, 116). Mit der Wittgenstein-Lektüre im Hintergrund wird der Aktivitätsgrad des Betrachters unterschiedlich bewertet: Während Krämer das aktive, tätige Umgehen mit der angeschauten Konfiguration betont, steht in David Lauers Auslegung stärker der Widerfahrnischarakter im Vordergrund, was aber nicht Passivität bedeutet. Für Lauer (2008) gehört das Kippbild zur Gattung der anamorphotischen Darstellungen, wenngleich es einen Grenzfall darstellt. Er unterscheidet fünf Arten von Anamorphosen und nennt für die Bestimmung und Differenzierung zwei Parameter: den einzunehmenden Betrachterstandpunkt 
und die (Wieder-)Erkennbarkeit des Dargestellten (bzw. die doppelte Lesbarkeit). »Beim klassischen Vexierbild aber sind die zwei Betrachterstandpunkte, denen sich auf dem Bild unterschiedliche Inhalte zeigen, gewissermaßen in einer einzigen Raumstelle verschmolzen. Sie sind, anders als bei der Anamorphose im engen Sinne, nur noch in einem übertragenen Sinne unterschiedliche Blickpunkte, aber doch unterschiedliche Blickwinkel oder Blickweisen: Die Anweisung für den, der das Bild entschlüsseln will, lautet nicht mehr >Stell dich hier hin!<, aber immer noch `Schau es so an!< Der richtige Blick muss auch hier gefunden werden, und bei der Suche nach ihm sind weiterhin Körperhaltungen im Spiel, auch wenn sie bis auf ein Minimum reduziert werden können. Am Ende mag es sich nur noch um das Schräglegen des Kopfes handeln, das Zusammenkneifen eines Auges oder um eine bestimmte Art, den Blick über die Darstellung gleiten zu lassen.« (Lauer 2008, 234). Etwas als etwas zu sehen und dann als etwas anderes, d.h. das Phänomen des Aspektwechsels, verdeutlicht, dass Wahrnehmung per se aspekthaft sei, so Lauer. »Wittgenstein unterscheidet daher zwischen dem >Aufleuchten und dem sstetigen Sehen eines Aspekts،, oder auch zwischen sakuter und schronischer Aspektwahrnehmung« (2008, 235-236).

Auch Whitney Davis greift Diskussionen rund um Kippbilder auf, wie sie in der Gestaltpsychologie und Philosophie an Figuren mit perspektivischer Ambivalenz (vgl. Albers) oder doppelgestaltigen (vgl. Hasen-Entenkopf; s. o.) sstatischen Zeichnungen entwickelt wurden. Davis (2011) verbindet in seinem Vorschlag einen Wahrnehmungsreflex erster Ordnung als natürliches Sehen (Sehen-als) mit einem intentionalen visuellen Sich-Erarbeiten weiterer höherer Ordnungen bzw. Rekursionen (Sehen-als-als-...). Während ersteres den Vorgang des »imaging « darstellt, involviert letzteres »reimaging« ein »imagining « und wird mit der »radical pictoriality « in Verbindung gebracht. Dies äußert sich etwa darin, dass sich angesichts des Hasen-Entenkopfs unwillkürlich das eine oder das andere einstellt (als), dann aber weitere Konkretionen dazugedacht werden können: Man erwirkt dann beispielsweise allein den Eindruck des Hasen (als-als) oder auch den schwarzen Hasen im Schlaglicht (als-als-als), etc. (vgl. Davis 2011, 199, Tabelle 3).

Es liegt nahe, die vorliegende Diskussion des Beispiels von Signers Video im Sinne Davis' als Sehen-als-als aufzufassen. Während sich die klassischen Beispiele (in dieser Stufe) gemeinhin auf zweifache Weise interpretieren lassen, führt Signers Arbeit - die wir als dynamisches Äquivalent zu den papierenen Kippbildern ansehen können - mindestens drei verschiedene Raumkonfigurationen vor, ${ }^{13}$ und zwar nacheinander. Bei Heufieber handelt es sich somit eigentlich nicht um ein Verschränken verschiedener Ansichten, die theoretisch immer schon simultan erkennbar wären. Darin könnte ein Unterschied zu Davis' Position liegen: Die Reinterpretationen (Umschwünge wie Umkeh-

13 Die Relation zwischen Innen- und Außenraum wurde hier nicht weiter ausgebaut. 
rung, Distanzierung bzw. Miniaturisierung) sind meines Erachtens nicht zu jedem Zeitpunkt nach Belieben möglich, sondern bedürfen einer bildlichen Heranführung. Hier spielt die manifeste Bewegung des Videobildes und der gezeigten Vorgänge als Spezifikum eine wichtige Rolle. Wahrscheinlich hängt damit zusammen, dass eine gewisse Überraschung vonstatten geht, was gegen ein rein willentliches Ummodellieren des ersten Sinneseindrucks spricht. Unmerklich zeitigt sich in der Betrachtung etwas (eine Bewegung), das zu einem schlagartigen Raumumschwung führt, ohne dass man sagen könnte, wann dies sbegonnen hat. Verblüffenderweise erkennt man eine zu vermutende Transienzphase als solche nicht, sondern konstatiert sie nur retrospektiv. Als Betrachter zieht man diesbezüglich immer nur die Konsequenzen: Plötzlich erscheint einem eine andere Interpretation der Situation plausibler - der Gesichtseindruck hat sich umorganisiert.

\section{Schluss}

Wenn häufig gesagt wird, Roman Signers Arbeiten seien `Zeitskulpturen<, die er über Video dokumentiert, scheint es sich bei Heufieber eher um eine sbewegte Raumplastikı zu handeln, die über die Temporalitätsgestaltung zustande kommt, aber den Betrachter - fundamentaler als üblich - ins Konzept integriert, ihn als konstitutiven Teil ansieht. Der Garten zeigt ostentativ, was bei Signer erst durch den Rezipienten geleistet werden muss. Bei Signer sind wir selber in der Position des Kindes und können - trotz oder gerade wegen des single shot erkennen, dass wir den Raum konfigurieren - gleich mehrmals hintereinander auf verschiedene Weise. Der Raum, um den es in den Beispielen geht, ist ein dargestellter und zugleich erlebter. Beide Aspekte stehen in den diskutierten Arbeiten jedoch unterschiedlich zueinander: Während bei Der Garten der dargestellte Raum durch das (dargestellte) Erleben der Protagonistin perspektivisch affiziert wird, provoziert in Heufieber der dargestellte Raum ein Rezeptionserleben, das ihn zeitlich differenziert als unterschiedliche Raumsituationen wahrnehmbar macht.

\section{Literatur}

Arnheim, Rudolf (1965): Kunst und Sehen. Eine Psychologie des schöpferischen Auges. Berlin: De Gruyter.

Beier, Thaddeus/Neely, Shawn (1992): Feature-Based Image Metamorphosis. Computer Graphics 26, 2, 35-42.

Bergson, Henri (1991 [1896]): Materie und Gedächtnis. Eine Abhandlung über die Beziehung zwischen Körper und Geist, übers. v. Julius Frankenberger. Hamburg: Meiner.

Bergson, Henri (1907/1912): Schöpferische Entwicklung. Jena: Eugen Diederichs.

Boehm, Gottfried (1994): Die Wiederkehr der Bilder. S. 11-38 in: ders. (Hrsg.), Was ist ein Bild? München: Fink. 
Bucher, François (1961): Josef Albers: Trotz der Geraden: eine Analyse seiner graphischen Konstruktionen. Bern: Benteli.

Butler, Brian D. (2008): Wunsch. S. 15-19 in: ders. (Hrsg.), Roman Signer. Sculpting in Time. Bielefeld: Kerber.

Davis, Whitney (2011): The Archaeology of Radical Pictoriality. S. 191-218 in: Richard Heinrich / Elisabeth Nemeth / Wolfram Pichler / David Wagner (Hrsg.), Image and Imaging in Philosophy, Science and the Arts. Frankfurt a. M./Lancaster/Paris: ontos.

Drühl, Sven (2000): Tamás Waliczky - Der Zeitkünstler. Kunstforum International 151, 137-145.

Duguet, Anne-Marie (1996): Tamás Waliczky. o. S. [S. 3-5] in: Tamás Waliczky - Trilogy. Tokyo: NTT.

Good, Paul (2002): Zeit Skulptur. Roman Signers Werk philosophisch betrachtet, Unikate. Zürich / Köln: Walter König.

Good, Paul (2009): Roman Signer. Härtetest des Schönen. Köln: DuMont.

Hansen, Mark B. N. (2001): Seeing with the Body: The Digital Image in Postphotography. Diacritics 13, 4, 54-84.

Hansen, Mark B. N. (2004): New Philosophy for New Media. Cambridge/London: MIT.

Heßler, Martina/Mersch, Dieter (2009): Bildlogik oder Was heißt visuelles Denken? S. 8-62 in: dies. / ders. (Hrsg.), Logik des Bildlichen. Zur Kritik der ikonischen Vernunft. Bielefeld: transcipt.

Hock, Howard S. (1984): Christina's World: Imaginary Perspectives and the Encoding of Spatial Alignment Relations. S. 167-187 in: W. Ray Crozier / Antony J. Chapman (Hrsg.), Cognitive Processes in the Perception of Art, Amsterdam/New York/Oxford: NorthHolland.

Hünnekens, Annette (2007): Die Zukunft des Video-Clip: EyeWire Motion and Picture Tracking. Zur Wahrnehmungsgeschichte und Ästhetik dynamischer Bilder. S. 108-139 in: Gerhard Johann Lischka (Hrsg.), ZappingZone, Bern: Benteli.

Kluszczyński, Ryszard W. (2003): screens/images/worlds transformations of seeing. Art Inquiry 5, 14, 87-104.

Krämer, Sybille (2009): Operative Bildlichkeit. Von der >Grammatologie $<$ zu einer >Diagrammatologie ? Reflexionen über erkennendes `Sehen ler/Dieter Mersch (Hrsg.), Logik des Bildlichen. Zur Kritik der ikonischen Vernunft. Bielefeld: transcript.

Lauer, David (2008): Anamorphotische Aspekte. Wittgenstein über Techniken des Sehens. S. 230-244 in: Kyung-Ho Cha / Markus Rautzenberger (Hrsg.), Der entstellte Blick. Anamorphosen in Kunst, Literatur und Philosophie. München: Fink.

Luhmann, Niklas (1995): Die Kunst der Gesellschaft. Frankfurt a. M.: Suhrkamp.

Luhmann, Niklas (1997): Die Autonomie der Kunst. S. 177-190 in: Institut für soziale Gegenwartsfragen Freiburg i.Br./Kunstraum Wien (Hrsg.), Art \& Language \& Luhmann. Wien: Passagen.

Luhmann, Niklas (2008): Wahrnehmung und Kommunikation anhand von Kunstwerken. S. 246-258 in: Niels Werber (Hrsg.), Niklas Luhmann. Schriften zu Kunst und Literatur. Frankfurt a.M.: Suhrkamp.

Massumi, Brian (1998): Sensing the Virtual, Building the Insensible. Architectural Design $68,5-6,16-24$.

Massumi, Brian (2007): The Thinking-Feeling of What Happens. S. 70-91 in: Joke Brouwer/Arjen Mulder (Hrsg.): Interact or Die!, Ausst. Kat. DEAF 2007, Rotterdam: V2_ Publishing / Nai Publishers.

Morse, Margaret (1997): Tamás Waliczky. The Garden 21 ${ }^{\text {st }}$ century amateur film 1992. Computeranimation. S. 26-29 in: Institut für Bildmedium/ZKM (Hrsg.), Hardware Software Artware. Die Konvergenz von Kunst und Technologie, Kunstpraktiken am ZKM-Institut für Bildmedien 1992-1997. Karlsruhe: ZKM/Ostfildern: Cantz.

Müller, Axel (1997): Die ikonische Differenz. Das Kunstwerk als Augenblick. München: Fink. 
Röd, Wolfgang (1991): Erfahrung und Reflexion. Theorien der Erfahrung in transzendentalphilosophischer Sicht. München: Beck.

Signer, Roman (2008): o. T. [Buchcover] in: Jürg Nänni: Visuelle Wahrnehmung/Visual Perception. Sulgen/Zürich: Niggli.

Spuybroek, Lars (1997): Flüssige Form. Arch+ 138, 70-75.

Szepesi, Anna (2001): Tamás Waliczky: Der Wald. S. 111-119 in: ZKM Karlsruhe (Hrsg.), artintact komplett, Bd. 1-5, 1994-1999. Stuttgart: Cantz.

Van den Bosch, Paula (2000): An object falls to pieces in a way that is unprecedented, unforeseen and often of inconceivable beauty. S. 5-10 in: o. A. (Hrsg.), Roman Signer Works 1971-2000, Maastricht: Bonnefantenmuseum.

Van den Bosch, Paula (2006): Paula van den Bosch in conversation with Roman Signer. S. 7-42 in: Gerhard Mack/ dies. / Jeremy Millar (Hrsg.), Roman Signer. New York: Phaidon.

Waliczky, Tamás (1995): Der Garten (ein Amateurfilm des 21. Jahrhunderts). S. 354-362 in: Klaus Peter Dencker (Hrsg.), Interface 2: Weltbilder - Bildwelten. Computergestützte Visionen, Hamburg: Hans-Bredow-Institut.

Waliczky, Tamás (o.J.): Projektbeschreibung. http://www.c3.hu/scca/butterfly/Waliczky/ project.html (2.12.2011).

Withers, Rachel (2007): Roman Signer: Zeitschmied. S. 9-51 in: Friedrich Christian Flick Collection (Hrsg.), Roman Signer. Künstlermonographien - Friedrich Christian Flick Collection, Band 7. Köln: DuMont.

Wittgenstein, Ludwig (1984a): Bemerkungen über die Philosophie der Psychologie, Bd. I (Werkausgabe, Bd. 7). Frankfurt a. M.: Suhrkamp.

Wittgenstein, Ludwig (1984b): Letzte Schriften über die Philosophie der Psychologie, Bd. III (Werkausgabe, Bd. 7). Frankfurt a.M.: Suhrkamp.

Wittgenstein, Ludwig (1984c): Bemerkungen über die Philosophie der Psychologie, Bd. II (Werkausgabe, Bd. 7). Frankfurt a.M.: Suhrkamp.

Wittgenstein, Ludwig (2001): Philosophische Untersuchungen, Teil II, hg. v. Joachim Schute. Frankfurt a. M.: Suhrkamp.

Dr. Inge Hinterwaldner

Kunsthistorisches Seminar, Universität Basel

St. Alban-Graben 8, CH- 4051 Basel

inge.hinterwaldner@unibas.ch 
Copyright of Soziale Systeme is the property of Lucius \& Lucius Verlagsgesellschaft mbH and its content may not be copied or emailed to multiple sites or posted to a listserv without the copyright holder's express written permission. However, users may print, download, or email articles for individual use. 